\title{
Diagnostic Challenge \\ Dyspnea and Abdominal Pain in an Alcoholic
}

DOI: http://dx.doi.org/10.5915/17-2_3-12762

Qaisar Rasheed, M.D.

Bashir A. Chaudhary, M.D., F.C.C.P.

William A. Speir, M.D., F.C.C.P.

\section{History}

A 42 year old black male presented with the complaints of upper abdominal pain and shortness of breath. He had previous hospitalizations for alcoholism and pancreatitis. Physical examination revealed a cachectic man with BP $110 / 70 \mathrm{mmHg}$; temperature $37.2^{\circ} \mathrm{C}$; respiration $18 / \mathrm{min}$; and pulse $80 / \mathrm{min}$. Lung fields were clear except for diminished breath sounds in the right base. Abdominal examination was unremarkable.

White blood cell count was $8200 / \mathrm{cu}$ mm with $68 \%$

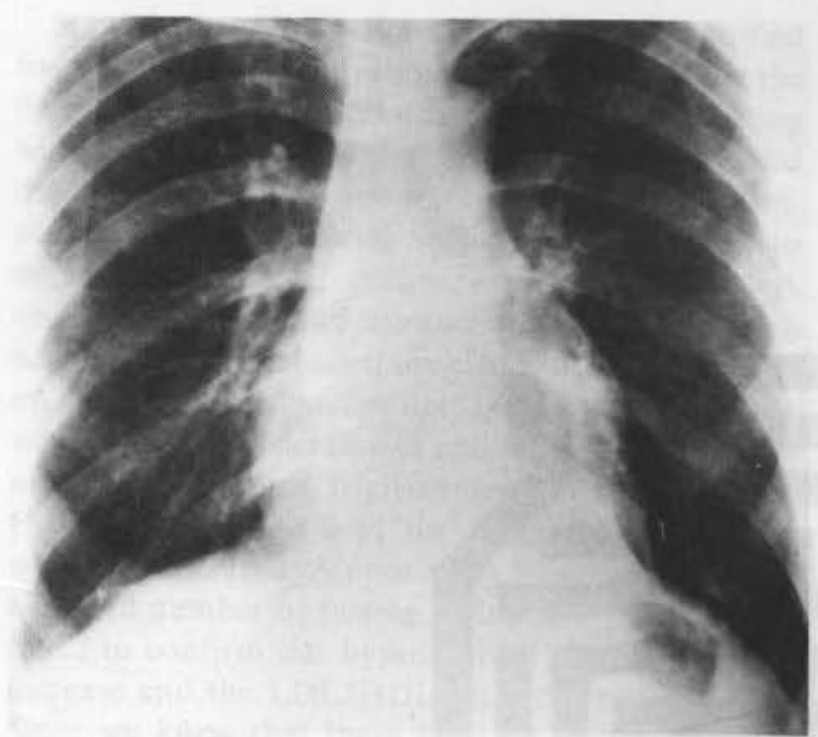

Figure 1: Chest radiograph at the time of admission.

From the Section of Pulmonary and Critical Care Medicine, Department of Medicine, Medical College of Georgia, Augusta, Georgia Address all correspondence to

Bashir A. Chaudhary, M.D.

Section of Pulmonary and Critical Care Medicine, Department of Medicine, Medical College of

Georgia, Augusta, Georgia 30912 polymorph-nuclear cells and $25 \%$ lymphocytes. His hemoglobin was $13.6 \mathrm{G} \%$ and serum amylase was 442 I.U./ml. His chest roentgenogram showed small bilateral pleural effusion, a prominence on the left cardiac border in the region of left atrial appendage, increased density at the left cardiophrenic region, and a soft tissue density behind the heart obscuring the left hemidiaphragm (Fig. 1). Upper GI series revealed anterior and lateral displacement of distal esophagus (Fig. 2). Pleural fluid obtained by thoracentesis showed an amylase level of $1338 \mathrm{I} . \mathrm{U} . / \mathrm{ml}$.

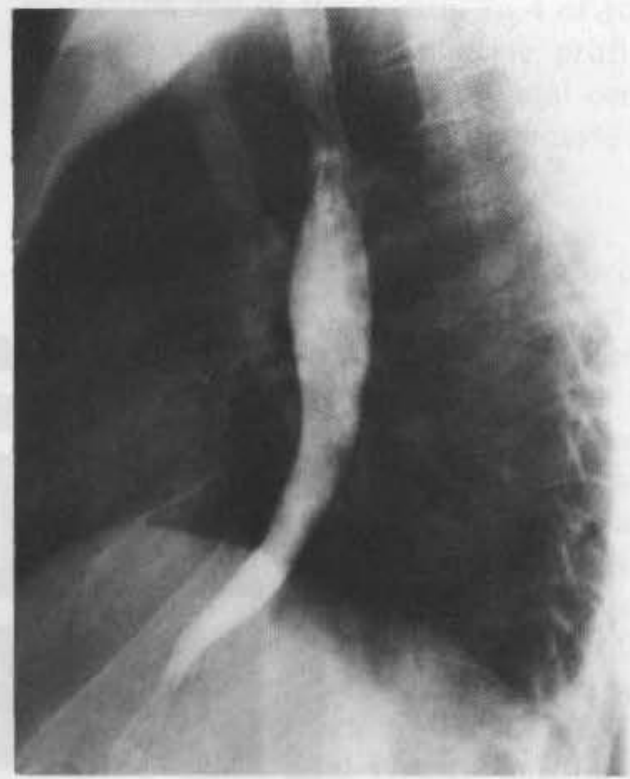

Figure 2: Upper GI series. 


\section{Pancreatic Pseudocyst with Mediastinal Extension}

A pre-operative diagnosis was made by CT scan, which revealed a retrogastric cystic mass in the lesser sac extending into the posterior mediastinum presumably through the esophageal hiatus (Fig 3). The diagnosis was consequently confirmed by surgical exploration. The pseudocyst was drained internally via cystogastrostomy and the patient rapidly improved. Postoperative chest roentgenogram showed clearing of the soft tissue density in the left cardiophrenic region and clearance of the prominence along the left cardiac border (Fig. 4).

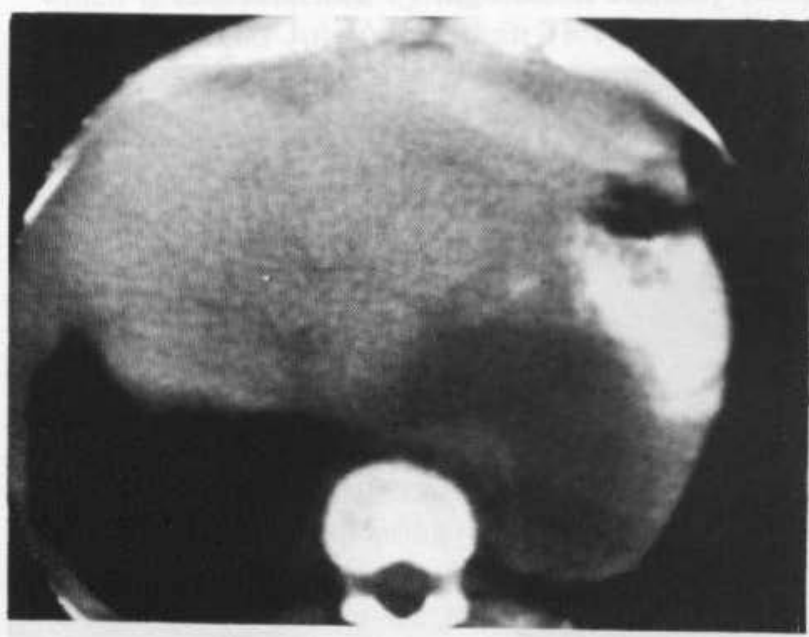

Figure 3: CT scan showing a retrogastric cystic mass extending into posterior mediastinum.

Mediastinal extension is an uncommon complication of pancreatic pseudocyst. It should be suspected in patients with a mediastinal mass and an associated history of pancreatitis or alcoholism.

Many radiologic abnormalities have been described. The usual portal of entry is either the esophageal or aortic hiatus. Hence, pancreatic pseudocysts usually occur inferiorly in the posterior mediastinum. Pleural effusion is frequently present; however, it is not helpful in the diagnosis, since it is also common in uncomplicated abdominal pancreatic pseudocyst. Segmental atelectasis and pericardial effusion are other uncommon roentgenologic findings. Perhaps the most typical radiographic finding is anterior and lateral displacement of the lower esophagus and stomach on the upper GI series. Though ultrasound is useful in the detection of pancreatic pseudocyst, it may not demon-

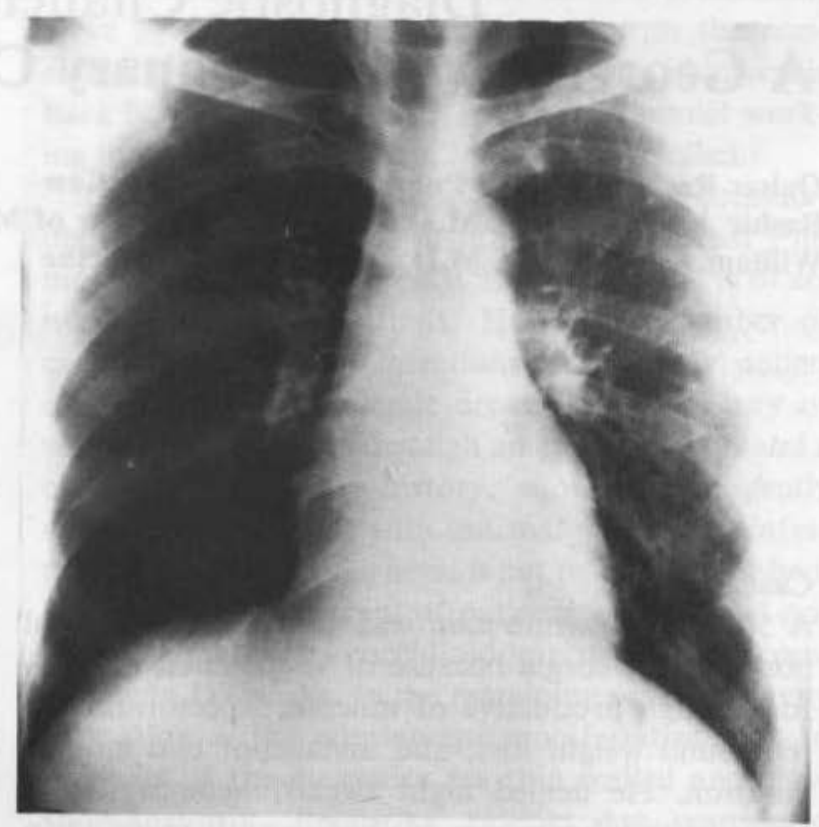

Figure 4: Chest radiograph after surgery showing clearing of the density.

strate the superior extension of the pseudocyst into the mediastinum because of the overlying boney sternum and air-filled lung. CT Scan is the best available means for the diagnosis of mediastinal pancreatic pseudocyst. ${ }^{2}$ It is also helpful in accurately delineating the pseudocyst for proper operative management.

A correct diagnosis of the mediastinal pancreatic pseudocyst is essential for proper surgical treatment, since it is best managed without thoracotomy. Abdominal exploration with internal drainage is the standard surgical procedure.

\section{References}

1. Kirchner SG, Heller RM, Smith CW: Pancreatic pseudocyst of the mediastinum. Radiology 1977 123:37-42.

2. Owens GR, Arger PH, Mulhern CB Jr, Coleman BG, Gohel V: CT evaluation of mediastinal pseudocyst. J Comput Assist Tomogr 1980 4:256-259. 